OSLO-TP 2-99

\title{
SPIN AND STATISTICS FOR QUANTUM HALL QUASI-PARTICLES 中
}

\author{
Jon Magne Leinaas \\ Department of Physics, University of Oslo \\ P.O. Box 1048 Blindern, N-0316 Oslo, Norway
}

\begin{abstract}
In two space dimensions the possibilities of fractional spin as well as fractional statistics exist. I examine the relation between fractional spin and statistics for Laughlin quasi-particles in a two-dimensional electron system with spherical geometry. The relevance of this for quasi-particles in a planar system is discussed.
\end{abstract}

\section{Statistics and spin in two dimensions}

I would like to begin by reminding you of the fact that in two space dimensions there is a richer set of possibilities than in higher dimensions as far as statistics and spin of particles is concerned. Quantum statistics is determined by the symmetry of the wave function under interchange of particle coordinates, and in three and higher dimensions the corresponding symmetry group is the permutation group. However, when particle interchange is viewed as a continuous process under which the coordinates are changed, then the symmetry group in two dimensions is larger, it is the two-dimensional braid group rather than the permutation group [1]. An element of this group does not only specify the permutation of the particles, but also the windings of the particle trajectories under the interchange of the positions. In dimensions higher than two these windings can be disentangled, since only interchanges corresponding to different permutations of the particles are topologically distinct. This is not possible in two dimensions.

For particles on the plane the coordinates can be written as complex variables, $z=x+i y$, and for two particles the symmetry under interchange of the particle

\footnotetext{
${ }^{1}$ To appear in the proceedings of the conference Orbis Scientiae 1998, Fort Lauderdale, December $18-21$.
} 
positions can be expressed as

$$
\psi\left(e^{i n \pi}\left(z_{1}-z_{2}\right)\right)=e^{i n \theta} \psi\left(z_{1}-z_{2}\right),
$$

where only the relative coordinate has been written out explicitly. In this expression $n$ is the winding number of the particle trajectory in 2-particle space, and $\theta$ is the parameter that specifies the statistics. The symmetry follows from the assumption that all configurations which differ only by an interchange of the particle positions are physically indistinguishable. The wave function for these configurations should therefore differ at most by a phase factor. Also for more than two (identical) particles the symmetry factors have the form $\exp (\operatorname{in} \theta)$ and they define a one-dimensional representation of the braid group for the particles. In two dimensions $\theta$ is a free parameter, while in higher dimensions it is restricted to the values $\theta=0(\bmod 2 \pi)$ for bosons and $\theta=\pi(\bmod 2 \pi)$ for fermions. For values of $\theta$ different from these two the particles are said to satisfy intermediate or fractional statistics, and they are referred to as anyons.

Also spin is different in two dimensions. In three dimensions the intrinsic spin of a particle is associated with the rotation group $S O(3)$. It is regarded as the generator of rotations in the rest frame of the particle. As is well known, the unitary representations of the rotation group $S O(3)$ restrict the allowed values of the spin to integer or halfinteger multiples of $\hbar$. For particles in two dimensions the rotation group is reduced to $S O(2)$. This is a one-parameter group with unitary representations

$$
U(\phi)=e^{i \phi S / \hbar},
$$

where $\phi$ is the rotation angle. In this case there is no restriction on $S$, it can take any real valuet.

Thus, statistics as well as spin can be regarded as continuous variables in two dimensions. An obvious question to ask is whether these two variables are linked by some kind of spin-statistics relation. This question has previously been discussed in different ways, and we know from theoretical constructions that many simple explicit models of two-dimensional particles have such a relation. Here I will consider this question in connection with a concrete realization: quasi-particles in the fractional quantum Hall effect. These quasi-particles are believed, on one hand to be real physical realizations of anyons in a quasi two-dimensional electron system, on the other hand to be well described (in some cases) by simple many-electron wave functions. The question of spin and statistics of these quasi-particles can therefore be examined rather directly, and has been done so in the past. One specific study is due to Einarsson et. al. [2], and my talk is inspired by this paper and can be seen as a comment to their result.

\section{Spin-statistics relations}

Since we are considering a non-relativistic system, I would like to stress the point that we cannot expect to find a spin-statistics theorem that on general grounds gives

\footnotetext{
${ }^{2}$ I am here actually referring to representations of the covering group of $S O(2)$, which are the relevant ones for quantum mechanics.
} 
a strict relation between these two particle properties. After all we have a simple counter-example to the standard relation between spin and statistics: spinless fermions described by one-component anti-symmetric wave functions. In the context of nonrelativistic many-particle theory there seems to be no problems with such a construction, and this is so for particles in two as well as in three space dimensions. Nevertheless, as soon as one leaves the simple point particle description and makes explicit models where the spin as well as the statistics can be derived from more fundamental fields, the standard spin-statistics relation seems naturally to appear in three-dimensional systems while a linear extension of this relation appear in two dimensions. Let me just mention some examples from two dimensions.

A simple electromagnetic model of an anyon is an electric point charge $e$ with an attached magnetic flux $\phi$, that is confined to a small region around the charge. (The mechanism that binds the flux to the charge is not so important and neither is the detailed profile of the magnetic field surrounding the charge.) In addition to the Coulomb interaction between such charge-flux composites, there will be an AharonovBohm interaction between the charge of one composite and the flux of the other. When two composites are interchanged the latter gives rise to a phase factor that can be identified with the statistics factor. A simple calculation gives for the statistics parameter

$$
\theta=-\frac{e \phi}{\hbar c}
$$

There is an electromagnetic spin associated with a charge flux composite, due to the overlap of the electric and magnetic fields. Using the expression for electromagnetic angular momentum reduced to its two-dimensional form, we calculate the spin to be

$$
S=-\frac{1}{c} \int d^{2} r B \vec{r} \cdot \vec{E}=-\frac{e \phi}{2 \pi c}
$$

We note that the statistics parameter and the spin both are determined by the same quantity $e \phi$.

A second example is provided by soliton solutions in the $O(3)$ non-linear $\sigma$-model with a topological (Hopf) term [3]. In this case the strength of the topological term determines the spin as well as the statistics of the solitons. A third example is given by the particles described by a scalar field theory with Chern-Simons coupling [4]. The Chern-Simons field gives an explicit realization of fractional statistics in the form of an Aharonov-Bohm effect. It also affects the conserved angular momentum and thereby links the spin to the statistics of the particles.

In the examples referred to above (as well as in some other examples) the relation between spin and statistics has the simple form

$$
S=\left[\frac{\theta}{2 \pi}(\bmod 1)\right] \hbar .
$$

It coincides with the standard relation for bosons $(\theta=0)$ and fermions $(\theta=\pi / 2)$ and extends that linearly to all other values of the statistics parameter $\theta$. 
Even if the simple relation (5) is favoured by many anyon models, we do not have a clear specification of the general conditions under which the relation should be satisfied. There do exist, however, some general arguments for a less restrictive form of the spinstatistics relation that are based on the assumption that there exist both anyons and anti-anyons in the system under consideration. Let me briefly give the arguments for this generalized spin-statistics relation, since it is relevant for the quantum Hall quasi-particles.

We then assume that there exist fractional statistics particles of a type we denote by $p$ (with some unspecified statistics parameter $\theta$ ). There also exist another type of particles $\bar{p}$, that we consider as anti-particles to $p$. Since we are not considering a relativistic theory, we do not assume charge conjugation symmetry (symmetry between $p$ and $\bar{p}$ ). The important point is the assumption that a $p-\bar{p}$ pair can be created and annihilated inside the system. This means that all long range effects of a single particle are canceled by the corresponding effects of an anti-particle. This has consequences for statistics as well as for spin.

For a $p-\bar{p}$ pair there are no long-range Aharonov-Bohm effects. That means that the phase factor introduced by transport of another particle of type $p$ around the pair is the trivial factor 1 for a path far away from the two particles. If these two particles also are sufficiently far apart, the phase factor can be written as a product of one factor from each of the particles in the pair. We write this as

$$
\exp \left(2 i\left(\theta_{p p}+\theta_{p \bar{p}}\right)\right)=1 \text {. }
$$

We easily see that $\theta_{p p}$ is identical to the statistics phase $\theta$ of particles $p$. The other phase $\theta_{p \bar{p}}$ is sometimes referred to as a mutual statistics phase. It describes an AharonovBohm interaction between two non-identical particles $p$ and $\bar{p}$. Clearly we have a similar condition when a particle of type $\bar{p}$ is transported around the pair,

$$
\exp \left(2 i\left(\theta_{\bar{p} \bar{p}}+\theta_{\bar{p} p}\right)\right)=1 .
$$

The two conditions (6) and (7), and the symmetry relation $\theta_{\bar{p} p}=\theta_{p \bar{p}}$, mean that all phases can be expressed in terms of a single phase $\theta$,

$$
\begin{aligned}
& \theta_{\bar{p} \bar{p}}=\theta_{p p}=\theta(\bmod \pi) \\
& \theta_{\bar{p} p}=\theta_{p \bar{p}}=-\theta(\bmod \pi) .
\end{aligned}
$$

A rotation of the $p-\bar{p}$ pair by an angle $2 \pi$ also has to give rise to a trivial phase factor. We write this as

$$
\exp \left(2 \pi \frac{i}{\hbar}\left(L_{c m}+L_{r e l}+S_{p}+S_{\bar{p}}\right)\right)=1 .
$$

The orbital angular momentum has here been divided into a center-of-mass part $L_{c m}$ and a part determined by the relative motion, $L_{r e l} ; S_{p}$ and $S_{\bar{p}}$ are the intrinsic spins of the two particles. $L_{c m}$ has integer eigenvalues in multiples of $\hbar$, while the spectrum of $L_{r e l}$ is shifted due to the nontrivial phase $\theta_{p \bar{p}}$. The eigenvalues are $(n-\theta / \pi) \hbar, n=$ $0, \pm 1, \pm 2 \ldots$. With this inserted in (9) we get

$$
\frac{1}{2}\left(S_{p}+S_{\bar{p}}\right)=\left[\frac{\theta}{2 \pi}\left(\bmod \frac{1}{2}\right)\right] \hbar .
$$


This is the generalized spin statistics relation. It only involves the sum of the spins of the anyon and the anti-anyon. Even if these two spins are equal we note the relation is less restrictive than the relation (5). It does not exclude spinless fermions or bosons with half-integer spin.

\section{Anyons in the quantum Hall system}

The quasi-particles of the quantum Hall system are charged excitations in a 2-dimensional electron gas subject to a strong perpendicular magnetic field. In general the quasiparticles are fractionally charged and obey fractional statistics; they are charged anyons in a strong magnetic field. For special filling fractions of the lowest Landau level, $\nu=1 / m, m$ odd, there exist simple (trial) wave functions, originally introduced by Laughlin [5], for the ground state of the many-electron system as well as for the quasiparticle excitations. Expressed in complex electron coordinates, the (non-normalized) $N$-electron ground state has the form

$$
\psi_{m}\left(z_{1}, z_{2}, \ldots, z_{N}\right)=\prod_{i<j}\left(z_{i}-z_{j}\right)^{m} e^{-\frac{1}{4 \ell^{2}} \sum_{k=1}^{N}\left|z_{k}\right|^{2}}
$$

with $\ell=1 / \sqrt{\frac{\hbar c}{e B}}$ as the magnetic length, and $e B$ taken to be positive. The one quasihole state is

$$
\psi_{Z}^{q h}\left(z_{1}, z_{2}, \ldots, z_{N}\right)=\prod_{i=1}^{N}\left(z_{i}-Z\right) \psi_{m}\left(z_{1}, z_{2}, \ldots, z_{N}\right),
$$

with $Z$ as the position of the quasi-hole. Multi-hole wave functions are constructed in a similar way, with several prefactors of the form given in Eq.(12). For the oppositely charged quasi-electron Laughlin has suggested a wavefunction of the form

$$
\psi_{Z}^{q e}\left(z_{1}, z_{2}, \ldots, z_{N}\right)=\prod_{i=1}^{N}\left(\frac{\partial}{\partial z_{i}}-Z^{*}\right) \psi_{m}\left(z_{1}, z_{2}, \ldots, z_{N}\right) .
$$

Supported by general arguments, as well as numerical studies, the ground state and the quasi-hole state are believed to be very well represented by the wave functions (11) and (12) (in a homogeneous system). However there is an asymmetry between the quasi-hole and the quasi-electron, and one should note that there is not a similar strong evidence in favour for the quasi-electron wave function (13) $)^{3}$.

The form of the quasi-particle wave functions determine the fractional charge as well as their fractional statistics. This was demonstrated by Arovas, Schrieffer and Wilczek who calculated the Berry phases associated with shifts of the quasi-particle coordinates along closed curves [8]. Let me give a brief comment on this in general terms.

\footnotetext{
${ }^{3}$ For a recent discussion see Ref. [7].
} 
The wave functions for configurations with $M$ quasi-holes define a $M$ (complex) dimensional submanifold in the $N$-electron Hilbert space parameterized by the quasihole coordinates. A fractional statistics representation (or anyon representation) [9] of the system can be introduced in terms of wave functions defined on this manifold, $\psi\left(Z_{1}, Z_{2}, \ldots, Z_{M}\right)$. The $M$-dimensional manifold, on which the wave-functions are defined can be interpreted as the configuration space (alternatively as the phase space) of the (classical) $M$ quasi-hole system. In a low-energy approximation we may consider the system restricted to this space. The kinematics as well as the dynamics of the quasi-hole system are determined from the $N$-electron system by projection on the complex submanifold. In particular, the kinematics is determined from the geometry of the manifold, and the charge and the statistics appear as geometrically determined parameters.

The scalar product of the $N$-electron Hilbert space defines, by projection, a complex geometry in the $M$-dimensional quasi-hole space. It is expressed in terms of the Hermitian matrix

$$
\eta_{k l}=\left\langle D_{k} \psi \mid D_{l} \psi\right\rangle
$$

with

$$
D_{k}=\partial_{k}+i A_{k}, \quad A_{k}=i\left\langle\psi \mid \partial_{k} \psi\right\rangle \text {. }
$$

$|\psi\rangle$ denotes the $M$-quasi-hole state and $\partial_{k}$ is the partial derivative with respect to a set of real coordinates in the quasi-hole space. $A_{k}$ is the Berry connection defined by the set of quasi-particle states. The real (and symmetric) part of $\eta_{k l}$ determines a metric on the $M$ quasi-particle space

$$
g_{k l}=\operatorname{Re}\left\langle D_{k} \psi \mid D_{l} \psi\right\rangle
$$

while the imaginary (and anti-symmetric) part determines a symplectic form, that we identify as the "Berry magnetic field",

$$
b_{k l}=2 \operatorname{Im}\left\langle\partial_{k} \psi \mid \partial_{l} \psi\right\rangle=\partial_{k} A_{l}-\partial_{l} A_{k} .
$$

For a single quasi-hole the form of $\eta_{k l}$ is strongly restricted by translational and rotational invariance (in the limit $N \rightarrow \infty$ ) and by analyticity in the variable $Z$,

$$
\eta_{k l}=-\frac{b_{1}}{2}\left(\delta_{k l}+i \epsilon_{k l}\right)
$$

Here $b_{1}$ is a constant that can be expressed in terms of the the real magnetic field, $b_{1}=\frac{e^{*} B}{\hbar c}$, with the coefficient $e^{*}$ as the effective charge of the quasi-hole. A Berry phase calculation for a loop in the plane determines the flux of $b_{1}$ through this loop, and comparison with the real magnetic flux then gives the effective charge $e^{*}[8]$.

For a two quasi-hole state an expression similar to (18) is valid for $\eta_{k l}$, if this now refers to the relative coordinate of the two quasi-holes. In this case $b_{1}$ is replaced by a function $b_{2}(R)$ that depends on the relative distance $R$. For small $R$ the form of this function is determined by local properties of the quasi-holes. For large $R, b_{2}(R)$ is 
expected to approach rapidly the constant $\frac{1}{2} b_{1}$ when the quasi-holes are well localized objects. The flux of $b_{2}$ then has the form

$$
\int_{r<R} d^{2} r b_{2}(R)=\frac{1}{2} \pi R^{2} b_{1}-2 \theta,
$$

where $\theta$ is identified as the statistics parameter of the quasi-holes. Again this parameter can be determined by a Berry phase calculation, that measures the flux of $b_{2}(R)$ within a given radius.

Berry phase calculations based on the quasi-hole wave function (12) gives $e^{*}=$ $-e / m$ for the charge and $\theta=-\pi / m$ for the statistics parameter, with $e$ as the electron charge [8]. For the quasi-electron wave function (13) one cannot derive the results so easily [6], but the expected results for the physical quasi-electron is $e^{*}=e / m$ and $\theta=2-\pi / m$, as determined from general reasoning and numerical studies [10].

Whereas charge and statistics can be determined geometrically, in terms of Berry phases associated with closed curves of one and two quasi-particles, the spin cannot be determined quite as easily. However, as pointed out by Einarsson [11] and Li [12] there is a way to derive spin from Berry phases, provided the particles move in a curved space. If the spin can be viewed as a three-dimensional spin constrained to point in the direction orthogonal to the two-dimensional surface, there will be a contribution to the Berry phase when transporting the quasi-particle around a loop that is proportional to the product of the spin value and the solid angle traced out by the spin [13. This suggests the following form of the Berry magnetic field

$$
b_{1}=\frac{e^{*} B}{\hbar c}-\frac{S}{\hbar} \kappa,
$$

with $\kappa$ as the Gauss curvature and the coefficient $S$ as the spin. It is not obvious that calculations of Berry phases for quasi-holes will give a separation in two terms of this form, but if they do, the spin can be determined from the Berry phases. This is the assumption made in [2]. In this case a quantum Hall system with the geometry of a sphere is considered. One should note that in this case the magnetic field $B$ as well as the curvature $\kappa$ are constants. That means that there is no clear distinction between the two contributions to the Berry phase in Eq.(20). However if the charge $e^{*}$ of the quasi-particle on the sphere is the same as the quasi-particle charge on the plane (which seems reasonable), then the second term can be separated from the first one and the spin can be determined.

\section{Quantum Hall states on the sphere}

In practice, to create a quantum Hall system with the geometry of a sphere can hardly be done. A radially directed magnetic field is then needed, and this means that a magnetic monopole should be found and placed at the center of the sphere. However as a theoretical construction a spherical Hall system can easily be created, and as first shown by Haldane such a geometry may conveniently be used in the study of certain aspects of the quantum Hall effect [14]. Also for numerical calculations it is convenient due to the lack of boundaries [10]. 
To have a consistent quantum description of the electrons in the monopole field, Dirac's quantization condition has to be satisfied,

$$
\frac{e \phi}{4 \pi \hbar c}=\frac{1}{2} N_{\phi},
$$

where $\phi$ is the total flux of the monopole field and $N_{\phi}$ is an integer. This means that the total magnetic flux through the sphere is quantized in units of the flux quantum $\phi_{0}=\frac{h c}{e}$,

$$
\phi=N_{\phi} \phi_{0},
$$

with $N_{\phi}$ as the number of flux quanta.

Laughlin states like (111),(12) and (13i) can be constructed on the sphere and can conveniently be expressed in terms of the coordinates $u=\cos (\theta / 2)$ and $v=$ $\sin (\theta / 2) \exp (i \phi)$, with $\theta$ and $\phi$ as the polar coordinates on the sphere. The form of the ground state is (in the Dirac gauge $e \vec{A}=e B \tan \frac{\theta}{2} \vec{e}_{\phi}$ )

$$
\psi_{m}=\prod_{i<j}\left(u_{i} v_{j}-u_{j} v_{i}\right)^{m}, \quad N_{\phi}=m(N-1),
$$

and this is non-degenerate, with all particles in the lowest Landau level, provided the number of electrons $N$ is linked to the number of flux quanta $N_{\phi}$ as indicated above. If one flux quantum is added, a hole state is created,

$$
\psi_{U V}^{q h}=\prod_{i}\left(V u_{i}-U v_{i}\right) \psi_{m}, \quad N_{\phi}=m(N-1)+1,
$$

with $(U, V)$ as the quasi-hole coordinates, and if one flux quantum is removed, a quasielectron state is created,

$$
\psi_{U V}^{q e}=\prod_{i}\left(V^{*} \frac{\partial}{\partial u_{i}}-U^{*} \frac{\partial}{\partial v_{i}}\right) \psi_{m}, \quad N_{\phi}=m(N-1)-1,
$$

now with $(U, V)$ as the quasi-electron coordinates.

For the quasi-hole state a detailed calculation of the Berry phase has been performed in Ref. [2], with a discussion of the different contributions. I will not repeat that here, let me rather show how the result concerning the spin can be derived directly from rotational invariance, without reference to Berry phases. This derivation is based on the assumption that the quasi-particle can be represented as a particle with charge $e^{*}$ in the monopole field.

For a single electron moving in a magnetic monopole field, the conserved angular momentum has the form

$$
\vec{J}=\vec{r} \times \vec{\pi}+\mu \overrightarrow{\hat{r}},
$$

with $\vec{\pi}$ as the mechanical momentum,

$$
\vec{\pi}=\vec{p}-\frac{e}{c} \vec{A}
$$


and

$$
\mu=-\frac{e \phi}{4 \pi c}
$$

as the component of the total angular momentum in the radial direction $\overrightarrow{\hat{r}}$. This spin can be identified as the electromagnetic angular momentum due to the overlap of the electric field of the charge with the magnetic monopole field. This radially directed spin is quantized due to the Dirac condition,

$$
\mu=\frac{1}{2} N_{\phi} \hbar
$$

and this quantization condition can alternatively be derived directly from the requirement of rotational invariance, i.e. from the condition that the operator $\vec{J}$ should generate unitary representations of the rotation group.

Thus, there are two invariants associated with the angular momentum,

$$
\vec{J}^{2}=j(j+1) \hbar^{2}, \hat{\vec{r}} \cdot \vec{J}=\mu,
$$

with the restriction

$$
j=|\mu|,|\mu|+1, \ldots .
$$

The smallest value of $j$ can be identified as corresponding to the lowest Landau level, and as on the plane, the mechanical part of the angular momentum then has its smallest value. For $N$ electrons the total angular momentum is the sum of the contributions from each electron,

$$
\vec{J}=\sum_{i=1}^{N} \vec{J}_{i}
$$

The ground state (23) is rotationally symmetric, with $j=0$, while the spin of the quasi-hole state (24) is $j=N / 2$.

In the anyon representation the quasi-hole is represented as a (single) charged particle in the monopole field. If we assume that it can be treated as a point particle, the angular momentum has the same form as for a single electron,

$$
\vec{J}=\vec{r} \times \vec{\pi}+\left(\mu^{*}+S\right) \overrightarrow{\hat{r}} .
$$

In this expression $\vec{r}$ is the quasi-hole coordinate and $\mu^{*}=-\frac{e^{*} \phi}{4 \pi c}$ is the radially directed electromagnetic spin. $S$ is a possible additional radially directed spin, an intrinsic spin of the quasi-hole. We note that such an additional spin in fact has to be added in order to preserve rotational invariance. If $e^{*}$ is taken to be identical to the charge $e / m$ of a quasi-hole in a planar system, then $\mu^{*}=N_{\phi} / 2 m$. This is in general not a half-integer, and the condition for rotational invariance is therefore not satisfied with $S=0$. The value of $S$ can be determined if we identify the anyon coordinates with the coordinates 
$(U, V)$ of the quasi-hole state (24). The spin component of this state in the $(U, V)$ direction is $N / 2$, and this gives the relation

$$
\frac{1}{2 m} N_{\phi}+S=\frac{1}{2} N
$$

With the number of flux quanta related to the electron number as indicated in Eq.(24) this gives the spin value

$$
S_{q h}=\frac{1}{2}-\frac{1}{2 m}=\frac{1}{2}+\frac{\theta}{2 \pi} .
$$

where $q h$ now labels the spin of the quasi-hole. This result for the spin is the same as the one determined by Berry phase calculations [12, 2]. We note that the spinstatistics relation given by (35) is not identical to the relation (5) indicated by the anyon models referred to at an earlier stage. There is an additional term $1 / 2$ that looks like a shift between the boson and fermion value of $\theta$. However, one should also note that the contribution from the intrinsic spin of the electrons has not been included here. For fully polarized electrons in the plane this contribution is $-1 / 2 m$. For large electron numbers, this contribution is presumably the same on the sphere. Thus, with all contributions included we get $S_{q h}=\frac{1}{2}-\frac{1}{m}=\frac{1}{2}+\frac{\theta}{\pi}$, and we still do not recover the relation (5). The only exception is for $m=1$, the case of a fully occupied lowest Landau level. The spin is then $-1 / 2$, in accordance with the standard spin-statistics relation.

The quasi-electron state (25) can be examined in a similar way. The spin component in the radial direction in this case has the opposite sign and there is also a change in the relation between the number of flux quanta and the electron number. The spin value now is

$$
S_{q e}=\frac{1}{2}-\frac{1}{2 m}=-\frac{1}{2}+\frac{\theta}{2 \pi}
$$

The contribution from the intrinsic spin of the electrons in this case is $1 / 2 m$, which gives the total spin $S_{q e}=\frac{1}{2}$. Also here the original spin-statistics relation is not satisfied.

However, the two expressions (35) and (36) show that the generalized spin-statistics relation is satisfied in the form

$$
\frac{1}{2}\left(S_{q h}+S_{q e}\right)=\frac{\theta}{2 \pi} .
$$

That is the case also when the contribution from the intrinsic spin of the electrons are included, since the contribution to the quasi-electron spin is the same, but with opposite sign as the contribution to the quasi-hole spin.

\section{Spin on the sphere - spin on the plane}

The spin values (35) and (36) are determined for quasi-particles on a sphere. What conclusion can we now draw concerning quasi-particles in a planar system? Is there a 
local spin associated with the quasi-particles with value identical to the one found on a sphere? The discussion we find in Ref. [2], and also the results found in a paper by Sondhi and Kivelson [15], do not support this conclusion' Thus, if their conclusions are correct, there is no simple relation between the spin of the quasi-particle on the sphere and a spin derived from the angular momentum of the electrons in a planar system. This is somewhat disappointing since the main motivation for putting the quasi-particles on the sphere, I assume, was to be able to visualize the quasi-particle spin, not to create the spin. The usual picture of the quasi-particle excitations is that they are strongly localized in space and that they have particle like properties with sharply defined quantum numbers such as charge, mass and possibly spin. If the quasiparticle spin determined on the sphere is not the same as the quasi-particle spin on the plane, that presumably means that it cannot be thought of as a local spin associated with the quasi-particle. The spin could in principle be due to a small renormalization of the charge of the quasi-particle when put on a sphere,

$$
e_{\text {sphere }}^{*}=e^{*}\left(1+\frac{m-1}{m N}\right),
$$

However, the $N$ dependence of the correction term does not seem to fit the picture of the quasi-particle as a strongly localized object.

Let me briefly discuss the question of the quasi-particle spin for a planar system. The normal component of the conserved angular momentum of an electron in a homogeneous magnetic field is

$$
J=(\vec{r} \times \vec{\pi})_{z}+\frac{e B}{2} r^{2}
$$

with $\vec{r}$ as a vector in the $(x, y)$-plane. The first term is the mechanical angular momentum of the circulating electron, whereas the second term can be interpreted as the electromagnetic spin (with an infinite $\vec{r}$-independent term subtracted).

For electrons in the lowest Landau level, the conserved angular momentum can be written in the form

$$
J=\hbar\left(-\int d^{2} r \rho(r)+\frac{1}{2 \ell^{2}} \int d^{2} r r^{2} \rho(r)\right),
$$

with $\rho$ as the particle density. The first term, the mechanical angular momentum is proportional to the particle number, since all electrons in the lowest Landau level carry one unit of (mechanical) angular momentum. The second term is the contribution from the electromagnetic angular momentum. It has the opposite sign of the first term and dominates this so that for all angular momentum eigenvalues the spin is non-negative.

The total angular momentum (40) diverges with the size of the system, the first term as the electron number $N$ and the second term as $N^{2}$. This is so for the ground state (11) as well as for the quasi-particle states (12) and (13). Clearly, if a local, finite spin should be associated with the quasi-particle, one has in some way to subtract the

\footnotetext{
${ }^{4}$ Somewhat surprisingly this is not seen as a problem in [2], with the explanation that the spin in the planar system does not have a dynamical significance.
} 
angular momentum of the ground state. A simple definition of the quasi-particle spin would be

$$
S_{q p}=\lim _{R \rightarrow \infty}\left(J_{q p}(R)-J_{0}(R)\right) .
$$

where $J_{q p}(R)$ is the total angular momentum of the quasi-particle state within a radius $R$ and $J_{0}(R)$ the angular momentum of the ground state within the same radius. The size of the electron system is here regarded as infinite. Even if these two terms diverge separately for large $R$, the difference should stay finite and give a well-defined value for the spin.

The first term of the angular momentum (40) gives a contribution to the quasiparticle spin (after the subtraction of the ground state spin) which is determined by the charge of the quasi-particle. The contribution is $\pm 1 / m$ with + for the quasi-hole and - for the quasi-electron. The second term is not so easy to determine as the first term, but in the paper by Sondhi and Kivelson [15] (where a similar definition of the quasi-particle spin is used), there is a discussion of the quasi-hole case. In this case the plasma analogy, introduced by Laughlin, can be applied. In the plasma analogy the square modulus of the quasi-hole wave function (12) is interpreted as the partition function of a Coulomb system consisting of $N$ free (unit) charges in a homogeneous neutralizing background, with the presence of an additional fixed charge of value $1 / m$ (the quasi-hole). The integrated particle number is then determined as the screening charge of this fixed charge, with the value $-1 / m$. Also the second moment of the particle number density, which is relevant for the second term of the angular momentum, can be related to the value of the charge. In fact, assuming that the conditions for "perfect screening" to be satisfied [9], there is a cancellation between the two terms of the the angular momentum so that the quasi-hole spin, as defined above, vanishes. This is the conclusion of Sondhi and Kivelson . With this conclusion it it difficult to see any connection between the physical spin of the quasi-hole state in the plane and the spin determined on the sphere. If the physical spin vanishes for any value of $m$ this in fact rules out any connection between the (physical) spin and the statistics parameter of the quasi-particles.

However, as a final point I would like to pose the question whether the conclusion concerning the spin, which is based on the use of the plasma analogy, is necessarily true, or whether another conclusion may be possible. Clearly, for a full Landau level, with $m=1$, the quasi-hole spin vanishes since the hole is created simply by removing an electron in a spin 0 state. For $m=3$ the situation is not quite as obvious and one has to refer to the situation in a one-component plasma with a $1 / 3$ charge screened by a plasma of integer charges. I am not able to judge the claim that the perfect screening condition is satisfied in this case, but I have noted with interest that in Ref. [16] one refers to a "basic belief" in the underlying assumption when the perfect screening sum rule is derived.

There is of course a way to avoid the reference to the plasma analogy. That is to make a straight forward calculation of the spin (41) of the planar system, and I will cite

\footnotetext{
${ }^{5}$ Sondhi and Kivelson also consider corrections to the spin due to the electromagnetic selfinteraction of the quasi-hole. Such corrections are important in order to give the correct value of the spin for the physical quasi-hole, but have not been taken into consideration here.
} 
some preliminary results for Monte-Carlo calculations performed by Heidi Kjønsberg for an electron system consisting of $N=100$ electrons. The numerical calculations reproduce values for the integrated quasi-hole spin $S_{q h}$, within a variable radius $R$ around the quasi-hole, which is placed at the center of the circular electron system defined by the Laughlin wave function.

Let me first give some values for the spin evaluated on the sphere, as given by Eq. (35). For $m=1$ the spin is 0 , for $m=3$ the spin is $1 / 3$ and for $m=5$ the spin is $2 / 5$, all spins expressed in units of $\hbar$.

The numerical results for the the planar system agree well with the value 0 for the $m=1$ state. However, for $m=3$ this is not the case. For values of the radius $R$ that lie between the size of the quasi-hole and the size of the full electron system, the results indicate instead a fairly stable value close to $1 / 3$, that agrees with the value found on the sphere. For $m=5$ the results are not so clear, due to larger finite size effects and also due to larger statistical fluctuations in the Monte Carlo calculations. Nevertheless, also here the results indicate a spin value different from 0 and possibly consistent with the value $2 / 5$.

So I would like to finish by referring to the question of the spin of the quasi-hole as an interesting one which deserves a further study. I feel that the situation in a sense would be more satisfying if the spin evaluated on the sphere could be identified as the physical spin of the quasi-particle also for a planar quantum Hall system. But such a conclusion would raise some new and interesting questions concerning the use of the plasma sum rules for the Laughlin states.

\section{Acknowledgments}

I appreciate the help of Heidi Kjønsberg who have performed the numerical calculations referred to in this paper. I am grateful to Hans Hansson and Anders Karlhede for several helpful comments and would like to thank their group at the Department of Physics, Stockholm University, for hospitality during a stay in February 1999.

\section{References}

[1] J.M. Leinaas and J. Myrheim, On the Theory of Identical Particles, Nuovo Cimento 37B (1977) 1.

[2] T. Einarsson, S.L. Sondhi, S.M. Girvin and D.P. Arovas, Fractional Spin for Quantum Hall Effect Quasiparicles, Nucl. Phys.B 441 (1995) 515.

[3] F. Wilczek and A. Zee, Linking Numbers, Spin and Statistics of Solitons, Phys. Rev. Lett. 51 (1983) 2250.

[4] T.H. Hansson, M. Rocek and I. Zahed, Spin and statistics in massive (2+1)dimensional QED, Phys. Lett. B 214 (1988) 475. 
[5] R. B. Laughlin, Anomalous Quantum Hall Effect: An Incompressible Quantum Fluid with Fractionally Charged Excitations, Phys. Rev. Lett. 50 (1983) 1395.

[6] H. Kjønsberg and J. M. Leinaas, On the anyon description of the Laughlin hole states, Int. Jour. Mod. Phys. A 12 (1997) 1975.

[7] H. Kjønsberg and J. M. Leinaas, Charge and Statistics of Quantum Hall QuasiParticles: A numerical study of mean values and fluctuations, preprint Oslo TP 1-99, cond-mat/9901266.

[8] D. Arovas, J. R. Schriffer and F. Wilczek, Fractional Statistics and the Quantum Hall Effect, Phys. Rev. Lett. 53 (1984) 722.

[9] R. B. Laughlin, Elementary Theory: the Incompressible Quantum Fluid, in The Quantum Hall Effect, eds. R. E. Prange and S. M. Girvin, Springer-Verlag, 1990.

[10] M. D. Johnson and G. S. Canright, Haldane fractional statistics in the fractional quantum Hall effect, Phys. Rev. B 49 (1994) 2947.

[11] T. Einarsson, Fractional statistics on compact surfaces, Mod. Phys. Lett B 5 (1991) 675.

[12] D.P. Li, The spin of the quasi-particle in the fractional quantum Hall effect, Phys. Lett. A 169 (1992) 82.

[13] M. B. Berry, Quantal phase factors accompanying adiabatic changes, Proc. R. Soc. Lond. A. 392 (1984) 45.

[14] F. D. M. Haldane, Fractional Quantization of the Hall Effect: A Hierarchy of Incompressible Quantum Fluid States, Phys. Rev. Lett. 51 (1983) 605.

[15] S.L. Sondhi and S.A. Kivelson, Long-range interactions and the quantum Hall effect, Phys. Rev. B. 46 (1992) 13391.

[16] M. Baus and J.-P. Hansen, Statistical mechanics of simple Coulomb systems, Phys. Rep. 59 (1980) 1. 